VoL. $76(2007) \quad[315-320]$

\title{
SIMPLE RINGS WITH INJECTIVITY CONDITIONS ON ONE-SIDED IDEALS
}

\author{
John Clark and Dinh VAN HuYnh
}

\begin{abstract}
This paper looks at simple rings which have right ideals satisfying various types of injectivity conditions. We characterise when a simple regular ring is right self-injective and show that if $R$ is a simple ring in which every right ideal is the direct sum of quasi-continuous right ideals then $R$ is either Artinian or a non-selfinjective right Goldie ring in which every right ideal is a direct sum of uniform right ideals.
\end{abstract}

\section{Definitions AND NOtation}

In what follows, rings are always associative with identity, and modules are unitary modules. For a module $M$ and a positive integer $n, M^{n}$ denotes the direct sum of $n$ copies of $M$ while $M^{(N)}$ denotes the direct sum of a countably infinite number of copies of $M$.

Recall that a module $M$ is called a $C S$ module or extending if every complement (closed) submodule is a direct summand, or equivalently, if every submodule of $M$ is essential in a direct summand. A ring $R$ is called a right $C S$ ring if the right $R$-module $R_{R}$ is CS. If the module $M^{n}$ is CS for every $n \in \mathbb{N}$, then $M$ is called finitely $\Sigma$-CS. Since its introduction thirty years ago by Chatters and Hajarnavis in [2], the CS condition has been studied extensively, features prominently in Mohamed and Müller's [13], and is the focus of the text [6] by Dung, Huynh, Smith and Wisbauer.

A module $M$ is called quasi-continuous if it is CS and whenever $M_{1}$ and $M_{2}$ are summands of $M$ for which $M_{1} \cap M_{2}=0$ then $M_{1} \oplus M_{2}$ is also a summand of $M$.

Moreover, a module $M$ is called continuous if it is CS and every submodule of $M$ which is isomorphic to a direct summand of $M$ is in fact a direct summand of $M$. As usual, we say that the ring $R$ is right continuous if the module $R_{R}$ is continuous. Any injective module is continuous and every continuous module is quasi-continuous but the converses fail in general (see $[13,6]$ ).

Next, given two modules $M$ and $N, M$ is said to be $N$-injective (or $M$ is injective relative to $N$ ) if, for each monomorphism $f: K \rightarrow N$ and homomorphism $h: K \rightarrow M$,

Received 11th April, 2007

The second author wishes to express his sincere thanks to the Department of Mathematics and Statistics, Otago University, for their generous support and warm hospitality during his visit there in 2005.

Copyright Clearance Centre, Inc. Serial-fee code: 0004-9727/07 \$A2.00+0.00. 
there is a homomorphism $g: N \rightarrow M$ such that $g f=h$. (By Baer's Criterion, a right $R$-module $M$ is injective precisely when it is $R_{R}$-injective.) If $M$ is $N$-injective and $N$ is $M$-injective, we say that $M$ and $N$ are mutually injective.

Finally, we recall that the ring $R$ is (von Neumann) regular if $a \in a R a$ for any $a \in R$.

\section{SiMPLE RINGS WITH MUTUALLY INJECTIVE ONE-SIDED IDEALS}

In this section, we shall investigate the structure of simple regular rings which are right CS.

We begin with an easy but effective lemma, modelled on the proof of Hart's [10, Theorem 1]. This has also appeared in [3], but we repeat its short proof here for the sake of completeness.

LEMMA 2.1. Let $A$ be any nonzero right ideal of a simple ring $R$. Then, for some $n \in \mathbb{N}$, there are elements $x_{1}, x_{2}, \ldots, x_{n} \in R$ such that $R_{R}=x_{1} A+x_{2} A+\cdots+x_{n} A$. As a consequence, $R_{R}$ is isomorphic to a direct summand of $A^{n}$.

Proof: Since $R$ is simple and $R A$ is a nonzero two-sided ideal of $R$, we have $R=R A$. Thus, for some $n \in \mathbb{N}$, there are elements $x_{i} \in R, a_{i} \in A$ for $1 \leqslant i \leqslant n$ giving $1=x_{1} a_{1}+x_{2} a_{2}+\cdots+x_{n} a_{n}$. It now quickly follows that $R=x_{1} A+x_{2} A+\cdots+x_{n} A$.

Furthermore, we have an epimorphism $\varphi: A^{n} \rightarrow R$ defined by setting $\varphi$ : $\left(a_{1}, a_{2}, \ldots, a_{n}\right) \mapsto x_{1} a_{1}+x_{2} a_{2}+\cdots+x_{n} a_{n}$ for all $a_{1}, a_{2}, \ldots, a_{n} \in A$. The projectivity of $R_{R}$ splits $\varphi$ and so $R_{R}$ is isomorphic to a direct summand of $A^{n}$, as claimed.

This allows us to show the following.

Proposition 2.2. Let $R$ be a simple ring. If $R$ has two nonzero mutually injective right ideals then $R$ is a right self-injective ring.

Proof: Let $A$ and $B$ be two nonzero mutually injective right ideals of $R$. By Lemma 2.1, there are $m, n \in \mathrm{N}$ for which $R_{R}$ is isomorphic to a direct summand of $A^{m}$ and to a direct summand of $B^{n}$. Then, by [1, Propositions 16.10 and 16.13], $A^{m}$ and $B^{n}$ are also mutually injective. Then, applying [1, Propositions 16.13 and 16.10$]$ once more, it follows from this and the summand properties of $R_{R}$ that $A^{m}$ is $R_{R}$-injective and so $R_{R}$ is $R_{R}$-injective, as required.

Now note that for a regular ring $R$, if $A$ is a right ideal isomorphic to a direct summand of $R_{R}$ then of course $A$ is principal and so generated by an idempotent (see [8, Theorem 1.1]). From this it follows that a regular, right CS ring is right continuous (see [8, Corollary 13.4]). We make use of this in proving the following result. Since, by [8, Corollary 13.20], any right continuous indecomposable regular ring is right self-injective, the equivalence of (i) and (iv) is already known, but here we provide an alternative proof in the case when $R$ is simple.

PROPOSITION 2.3. For a simple regular ring $R$ the following conditions are equivalent. 
(i) $R$ is right $C S$.

(ii) The right $R$-module $R^{(\mathrm{N})}$ is CS.

(iii) $R$ contains a nonzero right ideal $A$ such that $A$ is finitely $\Sigma$-CS.

(iv) $R$ is right self-injective.

Proof: (i) $\Rightarrow$ (iv). If $R_{R}$ is uniform then, since $R$ is regular, $R$ must be a division ring and we're done. Thus suppose that $R$ is not uniform. Then, since $R_{R}$ is CS, we have $R_{R}=A \oplus B$ for two nonzero right ideals $A$ and $B$ of $R$. However, as noted above, $R_{R}$ is right continuous and so, by [13, Theorem 3.16], $A$ and $B$ are mutually injective. Then, by Proposition 2.2, $R$ is right self-injective, as required.

(iv) $\Rightarrow$ (ii) follows from Dung and Smith's [7, Proposition 3] while (ii) $\Rightarrow$ (iii) is clear.

(iii) $\Rightarrow$ (i). By Lemma 2.1, $R_{R}$ is isomorphic to a direct summand of $A^{m}$ for some $m \in \mathbb{N}$. Hence $R_{R}$ is CS.

In [3, Question 3.2], we asked if, given a simple right Goldie ring $R$, is $R$ right finitely $\Sigma$-CS if $\left(R^{2}\right)_{R}$ is CS? We note that Proposition 2.3 shows that this has a positive answer if $R$ is a simple regular ring.

Remark 2.4. The conditions of Proposition 2.3 are not left-right symmetric. To see this, let $V$ be an infinite-dimensional vector space over a field $F$, set $Q=\operatorname{End}_{F}(V)$ and $M=\left\{f \in Q: \operatorname{dim}_{F}(f V)<\operatorname{dim}_{F}(V)\right\}$. Then $M$ is the largest proper two-sided ideal of $Q$ and so $Q / M$ is a simple regular ring. Next let $R$ be the maximal right quotient ring of $Q / M$. Then $R$ is a simple, regular, right self-injective ring. However, as noted in [8, Example 10.11] and Goodearl and Handelman's [9, p. 805], $R$ is not directly finite. Consequently, by [8, Theorem 9.29], $R$ is not left self-injective. (In fact, ${ }_{R} R$ is not even CS by Proposition 2.2 - this can also be seen from an old result of Utumi [15, Lemma 5.3] stating that if $R$ is both left and right CS then $R$ must be directly finite.)

We next provide some information on the projectivity of the right ideals in the rings of Proposition 2.3.

COROLlary 2.5. If $R$ is a simple, regular, right CS ring then $R$ is right $\aleph_{0}$ hereditary; that is, every countably generated right ideal of $R$ is projective. Consequently, if every right ideal of $R$ is also countably generated, then $R$ is semisimple Artinian.

Proof: Given a countably generated right ideal $A$ of $R$, there is an epimorphism $f: R^{(\mathrm{N})} \rightarrow A$. Since $R^{(\mathrm{N})}$ is a right CS module by Proposition 2.3 and $A_{R}$ is nonsingular, $\operatorname{ker}(f)$ is a summand of $R^{(\mathrm{N})}$ and so $A$ is projective, as required.

If in addition every right ideal of $R$ is countably generated then $R$ must be right hereditary. Then, since $R_{R}$ is injective by Proposition 2.3, every cyclic right $R$-module must also be injective. Thus $R$ is semisimple Artinian by an old result of Osofsky [14, Theorem]. 


\section{SIMPLE RINGS AND QUASI-CONTINUOUS RIGHT IDEALS}

In this section we consider simple rings in which each right ideal is a direct sum of quasi-continuous right ideals. Once again, Lemma 2.1 plays a key rôle.

We say that a ring is non-selfinjective if it is neither right nor left selfinjective.

THEOREM 3.1. Let $R$ be a simple ring such that every right ideal is the direct sum of quasi-continuous right ideals. Then either

(a) $R$ is a non-selfinjective right Goldie ring in which every nonzero right ideal is a direct sum of uniform right ideals or

(b) $R$ is Artinian.

Proof: We first show that $R$ contains a uniform right ideal.

Suppose to the contrary. Then we must have $\operatorname{Soc}\left(R_{R}\right)=0$. Moreover, by our hypothesis, if $A$ is a nonzero right ideal, we may write $A=\bigoplus_{i \in I} A_{i}$ where each $A_{i}$ is quasicontinuous and, by our assumption, each $A_{i}$ must be decomposable, say $A_{i}=C_{i} \oplus D_{i}$ where $C_{i}$ and $D_{i}$ are nonzero right ideals. Then, by [13, Theorem 2.13], $C_{i}$ and $D_{i}$ are mutually injective. Consequently, $C_{i}$ is $D_{i}^{n}$-injective and $D_{i}$ is $C_{i}^{m}$-injective for all $n, m \in \mathbf{N}$. Since, by Lemma $2.1, R_{R}$ is isomorphic to a direct summand of $C^{k}$ for some $k \in \mathbf{N}$, it follows that $D_{i}$ is (R-)injective. Similarly, $C_{i}$ is also injective. Thus $A_{i}$ is injective for each $i \in I$ and $R_{R}$ is also injective. Now we choose $A$ to be a maximal right ideal of $R$.

Then, if the index set $I$ is finite, $A_{R}$ will be injective and so a summand of $R_{R}$. Then any complement of $A_{R}$ in $R_{R}$ will be a minimal right ideal of $R$. This contradicts the fact that $\operatorname{Soc}\left(R_{R}\right)=0$.

Thus $I$ must be infinite. In this case, let $H, K$ be two infinite disjoint subsets of $I$ with $H \cup K=I$. Further set $U=\bigoplus_{i \in H} A_{i}$ and $V=\bigoplus_{i \in K} A_{i}$. Then, since $H$ and $K$ are infinite, $U$ and $V$ can not be direct summands of $R_{R}$. However their injective hulls $U^{*}$ and $V^{*}$, respectively, are summands of $R_{R}$ (since $R_{R}$ is injective). It follows that $U^{*} \neq U$ and $V^{*} \neq V$. Now write $R_{R}=U^{*} \oplus V^{*} \oplus W$ for some right ideal $W$ of $R$. Then, since $A=U \oplus V$ is a maximal right ideal, $W$ must be zero and this gives $R / A \cong\left(U^{*} / U\right) \oplus\left(V^{*} / V\right)$ where both $U^{*} / U$ and $V^{*} / V$ are nonzero. This contradicts the maximality of $A$.

This contradiction shows that $R$ has a uniform right ideal, as claimed. Then, by [10, Theorem 2], $R$ is right Goldie. Once again, let $A$ be a nonzero right ideal of $R$ and write $A=\bigoplus_{i \in I} A_{i}$ where each $A_{i}$ is a nonzero quasi-continuous right ideal. Then, since $R$ is right Goldie, the index set $I$ must be finite and we may assume that each $A_{i}$ is indecomposable and so uniform. Now, if there are distinct indices $i, j \in I$ for which $A_{i} \oplus A_{j}$ is not quasi-continuous, it follows from [13, Theorem 2.13] that $A_{i}$ and $A_{j}$ are not mutually injective. It is then straightforward to see that $R_{R}$ is not injective. 
We now show that $R$ is also not left selfinjective. Assume to the contrary that ${ }_{R} R$ is injective and let $n$ be the (finite) uniform dimension of $R_{R}$. If ${ }_{R} R$ has infinite uniform dimension, then by the left injectivity of $R$ we can produce a set of $n+1$ nonzero orthogonal idempotents $e_{i}$ in $R$. This in turn produces the right ideal $e_{1} R \oplus \cdots \oplus e_{n} R \oplus$ $e_{n+1} R$ in $R$, which is impossible since the uniform dimension of $R_{R}$ is $n$. Hence ${ }_{R} R$ has finite uniform dimension and so $R$ is left Goldie by [10]. This is a contradiction because $R$ is not right selfinjective. Thus $R$ is not left selfinjective. Hence, in this case, $R$ is a ring satisfying (a).

On the other hand, if there is a right ideal $A$ for which there are $i \neq j$ such that $A_{i} \oplus A_{j}$ is quasi-continuous, then $A_{i}$ and $A_{j}$ are mutually injective by [13, Theorem 2.13]. Hence $R$ is right self-injective by Proposition 2.2. It now follows that $R$ is also right Artinian and so satisfies (b) (see, for example, [12, Corollary 13.4]).

REMARK 3.2. Cozzens [4] has produced principal left and right ideal domains $D$ which are simple but not division rings. Thus, for any $k \in \mathbb{N}$, the ring $R=M_{k}(D)$ is a simple left and right Noetherian hereditary ring which is neither left nor right self-injective. It follows from [11] that every nonzero left (right) ideal of $R$ is CS and so is a direct sum of uniform left (right) ideals. This shows that $R$ is a ring satisfying (a) of Theorem 3.1.

In light of this remark we would like to ask:

QUESTION 3.3. Let $R$ be a simple ring such that every right ideal is the direct sum of uniform right ideals. If $R$ is not right self-injective and u. $\operatorname{dim}\left(R_{R}\right)>1$, is $R$ right Noetherian?

REMARK 3.4. A ring $R$ is said to be right pseudo-injective if, for any right ideal $A$ of $R$, any monomorphism $f: A \rightarrow R$ can be extended to an endomorphism of $R$. It follows from results of Dinh in [5] that any right Goldie right pseudo-injective semiprime ring is semisimple Artinian. However it is unknown as to whether every simple right pseudoinjective ring is right self-injective. (It is the case if we assume in addition that the ring is right $\mathrm{CS}$.)

\section{REFERENCES}

[1] F.W. Anderson and K.R. Fuller, Rings and categories of modules, (second edition) (Springer-Verlag, New York, 1992).

[2] A.W. Chatters and C.R. Hajarnavis, 'Rings in which every complement right ideal is a direct summand', Quart. J. Math. Oxford 28 (1977), 61-80.

[3] J. Clark and D.V. Huynh, 'A study of uniform one-sided ideals in simple rings', Glasgow Math. J. (to appear).

[4] J.H. Cozzens, 'Homological properties of the ring of differential polynomials', Bull. Amer. Math. Soc. 76 (1970), 75-79.

[5] H.Q. Dinh, 'A note on pseudo-injective modules', Comm. Algebra 33 (2005), 361-369. 
[6] N.V. Dung, D.V. Huynh, P.F. Smith and R. Wisbauer, Extending modules (Longman Scientific \& Technical, Harlow, 1994).

[7] N.V. Dung and P.F. Smith, ' $\sum$-CS-modules', Comm. Algebra 22 (1994), 83-93.

[8] K.R. Goodearl, Von Neumann regular rings, (second edition) (Krieger Publishing Company, Malabar, 1991).

[9] K.R. Goodearl and D. Handelman, 'Simple self-injective rings', Comm. Algebra 3 (1975), 797-834.

[10] R. Hart, 'Simple rings with uniform right ideals', J. London Math. Soc. Ser. 142 (1967), 614-617.

[11] D.V. Huynh, S.K. Jain and S.R. López-Permouth, 'Prime Goldie rings of uniform dimension at least two and with all one-sided ideals CS are semihereditary', Comm. Algebra 31 (2003), 5355-5360.

[12] T.Y. Lam, Lectures on modules and rings (Springer-Verlag, New York, 1999).

[13] S.H. Mohamed and B.J. Müller, Continuous and discrete modules (Cambridge Univ. Press, Cambridge, 1990).

[14] B.L. Osofsky, 'Rings all of whose finitely generated modules are injective', Pacific $J$. Math. 14 (1964), 645-650.

[15] Y. Utumi, 'On continuous rings and self injective rings', Trans. Amer. Math. Soc. 118 (1965), 158-173.

Department of Mathematics and Statistics

University of Otago

PO Box 56

Dunedin

New Zealand

e-mail: jclark@maths.otago.ac.nz
Department of Mathematics

Ohio University

Athens, OH. 45701

United States of America

e-mail: buynh@math.ohiou.edu 